\title{
SMARCB1/INI1 Is Diagnostically Useful in Distinguishing $\alpha$-Fetoprotein-producing Gastric Carcinoma from Hepatocellular Carcinoma
}

\author{
KUNIO MOCHIZUKI, MASATAKA KAWAI, TORU ODATE, IPPEI TAHARA, \\ TOMOHIRO INOUE, KAZUNARI KASAI, TADAO NAKAZAWA and TETSUO KONDO \\ Department of Pathology, School of Medicine, University of Yamanashi, Chuo, Japan
}

\begin{abstract}
Background/Aim: Switch/sucrose non-fermentable (SWI/SNF)-related, matrix-associated, actin-dependent regulator of chromatin subfamily $B$ member 1 (SMARCB1), also named integrase interactor 1 , is one of the core subunit proteins in the SWI/SNF ATP-dependent chromatin remodeling complex encoded at chromosomal position 22q11.2. Complete loss of SMARCBI expression has been reported in various malignant tumors. Immunohistochemistry has demonstrated that SMARCB1 mutation/inactivation correlates well with loss of nuclear SMARCB1 expression. This study investigated SMARCB1 expression in hepatocellular carcinomas (HCCs) and $\alpha$-fetoprotein (AFP)-producing gastric carcinomas by immunohistochemistry. For comparison, SMARCBI immunostaining in intrahepatic cholangiocarcinoma (ICC) was also performed. Materials and Methods: Thirty classical HCCs, 30 ICCs and 10 AFP-producing gastric carcinomas were analyzed. Results: Only one case of HCC had focal labeling of SMARCBI in the nuclei. Twelve cases of ICC were immunopositive for SMARCB1, with either focal or diffuse reactivity. All AFP-producing gastric carcinomas were immunopositive for SMARCB1 in the nuclei, and the reactivity was consistently diffuse. Conclusion: SMARCBI is a potential marker for distinguishing metastatic AFP-producing gastric carcinoma from $\mathrm{HCC}$.
\end{abstract}

Switch/sucrose non-fermentable (SWI/SNF) is an ATPdependent chromatin remodeling complex encoded at chromosomal position 22q11.2. SWI/SNF-related, matrixassociated, actin-dependent regulator of chromatin subfamily

Correspondence to: Kunio Mochizuki, MD, Ph.D., Department of Pathology, School of Medicine, University of Yamanashi, 1110 Shimokato, Chuo, Yamanashi, 409-3898, Japan. Tel: +81 552739529, Fax: +81 552739534, e-mail: kuniom@yamanashi.ac.jp

Key Words: SMARCB1, INI1, $\alpha$-fetoprotein, gastric carcinoma, hepatocellular carcinoma.
B member 1 (SMARCB1), also known as integrase interactor 1 , is one of the core subunit proteins in that complex (1). Interactions between SMARCB1 and various key proteins lead to tumor proliferation and progression through the p16retinoblastoma pathway, WNT signaling pathway, sonic hedgehog signaling pathway and polycomb pathway (1).

SMARCB1 is universally expressed in the nuclei of all normal cells and readily identified using immunohistochemistry (2). Complete loss of SMARCB1 protein expression can occur in malignant rhabdoid tumors, epithelioid sarcomas, pancreatic undifferentiated rhabdoid carcinomas, carcinomas of the sino-nasal or gastrointestinal tract, renal medullary carcinomas, epithelioid malignant peripheral nerve sheath tumors, myoepithelial tumors and extra-skeletal myxoid chondrosarcomas (1). Immunohistochemistry has demonstrated that SMARCB1 mutation/inactivation correlates with loss of nuclear expression of SMARCB1, and SMARCB1 immunostaining is commonly used by surgical pathologists to diagnose certain types of tumors (3). SMARCB1 loss, which is rare in colorectal adenocarcinoma, is a late event and is associated with a higher grade, larger tumor size, poorer survival, mismatch repair deficiency, and v-raf murine sarcoma viral oncogene homolog B1 (BRAF)V600E mutation (3).

There are few studies on SMARCB1 expression in hepatocellular carcinomas (HCCs) $(4,5)$. In addition, the architectural and cytological features of HCCs mimic $\alpha$-fetoprotein (AFP)-producing gastric carcinomas and expression of AFP $(6,7)$. AFP-producing gastric carcinoma has two distinct morphological subtypes, hepatoid and clear cell. Hepatoid carcinoma cells manifest the full spectrum of hepatocytic features. They resemble hepatocytes not only morphologically, but also by producing bile and synthesizing the same serum proteins such as AFP, $\alpha$-1-antitrypsin, and albumin (7). AFP-producing gastric carcinoma, characterized by an elevated serum AFP level, has an aggressive clinical behavior with marked vascular invasion and high rates of liver metastasis (8). We hypothesized that SMARCB1 immunohistochemistry might be useful for differentiating 
metastatic AFP-producing gastric carcinoma from HCC. In this study, SMARCB1 expression in HCCs and AFPproducing gastric carcinomas was investigated using immunohistochemistry. For comparison, intrahepatic cholangiocarcinomas (ICCs) were also enrolled.

\section{Materials and Methods}

Patients. Classical HCCs (30 cases), ICCs (30 cases) and AFPproducing gastric carcinomas (10 cases) were retrieved from routine surgical pathology files of the University of Yamanashi Hospital and Kofu-Kyoritsu Hospital. Two pathologists (K.M. and T.K.) blinded to the original pathological diagnosis independently reviewed hematoxylin and eosin-stained slides. The Research Ethics Committee of the Faculty of Medicine, University of Yamanashi approved this study (approval number: 1879).

Immunohistochemistry. Sections 4- $\mu$ m-thick were cut from formalinfixed, paraffin-embedded tissue blocks that were dewaxed and rehydrated. A representative slide from each case was stained immunohistochemically with primary antibodies to SMARCB1 (polyclonal, dilution 1:200; Sigma-Aldrich, St. Louis, MO, USA) and Alpha-1-Fetoprotein (polyclonal, dilution 1:2000; Dako, Glostrup, Denmark). Antigen retrieval was achieved through heat treatment: autoclaving at $120^{\circ} \mathrm{C}$ for $10 \mathrm{~min}$ in citrate buffer $(\mathrm{pH} \mathrm{6)}$ for SMARCB1. After inhibiting endogenous peroxidase, the primary antibodies were reacted. N-Histofine Simple Stain MAX PO (MULTI) (Nichirei Biosciences, Tokyo, Japan) was used with diaminobenzidine as the chromogen and hematoxylin as a light counterstain. Two pathologists simultaneously reviewed immunostained sections using a double-headed light microscope and graded the immunopositivity with the following scores: $0=0 \%$ positive cells; $1+=1 \%$ to $10 \%$ positive cells; $2+=11 \%$ to $50 \%$ positive cells; and $3+=51 \%$ to $100 \%$ positive cells. AFP-producing gastric carcinoma was defined as that having AFP production confirmed by immunohistochemistry in more than $1 \%$ of the tumor cells (8).

\section{Results}

Results are summarized in Table I. Conventional HCCs showed the following immunostaining patterns: 97\% scored 0 (Figure 1D), $0 \%$ scored 1, 3\% scored 2, and 0\% scored 3 . None of the non-cancerous hepatocyte nuclei from any cases expressed SMARCB1 (Figure 1B). The ICCs scored as follows: $60 \%$ scored $0,13 \%$ scored $1,23 \%$ scored 2 , and $3 \%$ scored 3 (Figure 1F). All of the AFP-producing gastric carcinomas scored 3 (Figure 1H).

\section{Discussion}

In 1998, positional cloning and sequence analysis of malignant rhabdoid tumors eventually identified mutations, deletions and other somatic alterations in the SMARCBl gene (1). Subsequently, aberrant expression of the SMARCB1 protein has been reported in various tumors having one of three patterns of aberrant SMARCB1 expression: complete loss, mosaic expression and reduced expression [reviewed in (1)].
Sun et al. demonstrated SMARCB1 expression by western blotting in 27 pairs of HCC tissues and their adjacent noncancerous tissues (5). They showed that SMARCB1 expression was preserved in 84 out of 149 HCCs. Furthermore, reduced SMARCB1 expression correlated strongly with a worse overall survival rate (5). We found only one case of $\mathrm{HCC}$ that had focal immunolabeling for SMARCB1. It was a moderately differentiated HCC consisting of cells with mild to marked nuclear pleomorphism and multinucleated cells. The discrepancy between our study and the previous study may be due to the different antibodies used for identifying SMARCB1 expression. Otherwise, we found a faint amount of SMARCB1 expressed in the nuclei of most HCC cells and normal hepatocytes.

All 10 AFP-producing gastric carcinomas were positive for SMARCB1. In contrast, $97 \%$ of the HCC samples were negative for SMARCB1. The specificity of SMARCB1 for distinguishing AFP-producing gastric carcinoma from $\mathrm{HCC}$ was $97 \%$, which makes SMARCB1 immunohistochemistry a useful tool for differentiating AFP-producing gastric carcinoma from HCC. Although AFP-producing carcinomas arise mainly in the stomach, sporadic cases have been reported in the esophagus, papilla of Vater, pancreas, and large bowel as well as in non-gastrointestinal organs such as lung, cervix, endometrium, renal pelvis, and urinary bladder (6). These tumors tend to behave aggressively, and the majority had metastasized to the liver at the time of diagnosis (6). AFP-producing gastric carcinoma with hepatoid morphology is diagnostically challenging when trying to identify hepatic metastasis (7). Hepatocyte paraffin 1 (Hep-Par 1) is a developed monoclonal antibody that reacts with a hepatocyte-specific epitope and has $82 \%$ sensitivity and $90 \%$ specificity for HCC (6). Terracciano et al. showed that Hep-Par 1 was immunonegative in all seven of the AFPproducing gastric carcinoma samples they tested (hepatoid adenocarcinomas) and in all but one liver metastasis (6). Ushiku et al. demonstrated that spalt-like transcription factor 4 is a sensitive marker for AFP-producing gastric carcinoma, and that it is especially useful in distinguishing hepatoid gastric carcinoma from HCC (specificity of 100\%) (7).

In our study, $40 \%$ of the ICC cases showed a mosaic pattern of SMARCB1 protein expression and $60 \%$ showed complete loss. Although originally described in association with malignant rhabdoid tumors in the pediatric population, the SMARCB1 loss pattern has been reported occasionally in tumors from other sites, including the uterus, pancreas and sinonasal tract (3). At these sites, as well as in the gastrointestinal tract, SMARCB1 loss was associated with aggressive behavior and poor survival (3). Therefore, SMARCB1 expression patterns in ICCs may relate to patient prognosis.

In conclusion, SMARCB1 is a potential biomarker for distinguishing metastatic AFP-producing gastric carcinoma from HCC. 

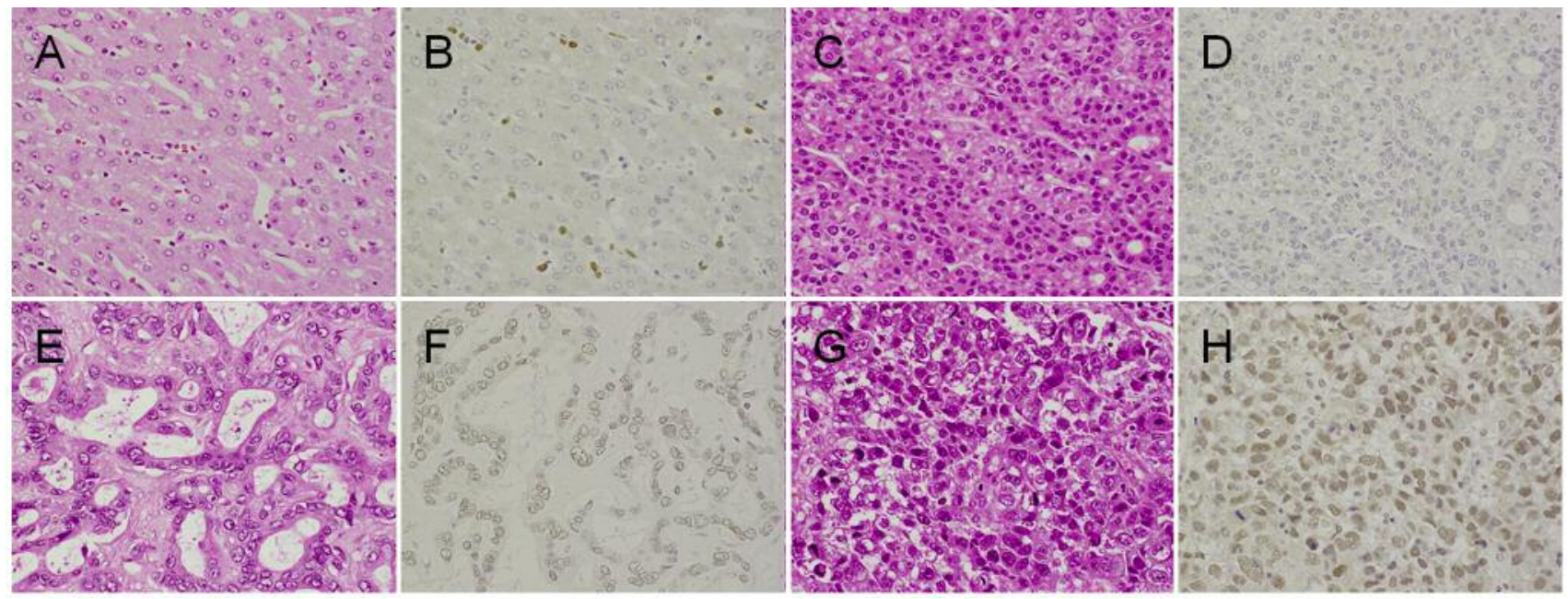

Figure 1. Representative immunohistochemical images of hematoxylin and eosin-stained ( $A, C, E$ and $G)$ and anti-switch/sucrose non-fermentablerelated, matrix-associated, actin-dependent regulator of chromatin subfamily B member 1 (SMARCB1)-stained (B, D, F and $H)$ tissue. Non-cancerous hepatocytes (A) exhibited no SMARCB1 immunoreactivity in the nuclei (B). Classical hepatocellular carcinoma (C) exhibited no SMARCB1 immunoreactivity $(D)$. Intrahepatic cholangiocarcinoma $(E)$ exhibited weak SMARCB1 immunoreactivity $(F)$. $\alpha$-fetoprotein-producing gastric carcinoma $(G)$ exhibited strong SMARCB1 immunoreactivity $(H)$. Original magnification $\times 400$.

Table I. Expression of switch/sucrose non-fermentable related, matrix-associated, actin-dependent regulator of chromatin subfamily B member 1 in hepatocellular carcinoma (HCC), intrahepatic cholangiocarcinoma (ICC) and a-fetoprotein (AFP)-producing gastric carcinoma (GC).

\begin{tabular}{lccccc}
\hline Primary tumor type & \multicolumn{3}{c}{ Immunohistochemical score, $\mathrm{n} *$} & \multirow{2}{*}{ Positive cases**(\%) } \\
\cline { 2 - 4 } & 0 & $1+$ & $2+$ & 1 & $3+$ \\
\hline HCC $(\mathrm{n}=30)$ & 29 & 0 & 7 & 0 & $1(3 \%)$ \\
ICC $(\mathrm{n}=30)$ & 18 & 4 & 0 & 1 & $12(40 \%)$ \\
AFP-producing GC $(\mathrm{n}=10)$ & 0 & 0 & 10 & $10(100 \%)$ \\
\hline
\end{tabular}

*Score $0: 0 \%$ reactive tumor cells; $1+: 1 \%$ to $10 \%$ reactive tumor cells; $2+: 11 \%$ to $50 \%$ reactive tumor cells; and $3+: 51 \%$ to $100 \%$ reactive tumor cells. **Any amount of reactivity was considered positive.

\section{Conflicts of Interest}

None of the Authors have any conflict of interest in regard to this study.

\section{Acknowledgements}

The Authors thank Ms. W. Iha for the technical support.

\section{References}

1 Kohashi K and Oda Y: Oncogenic roles of SMARCB1/INI1 and its deficient tumors. Cancer Sci 108: 547-552, 2017.
2 Hollmann TJ and Hornick JL: INI1-deficient tumors: Diagnostic features and molecular genetics. Am J Surg Pathol 35: e47-63, 2011.

3 Wang J, Andrici J, Sioson L, Clarkson A, Sheen A, Farzin M, Toon CW, Turchini J and Gill AJ: Loss of INI1 expression in colorectal carcinoma is associated with high tumor grade, poor survival, BRAFV600E mutation, and mismatch repair deficiency. Hum Pathol 55: 83-90, 2016.

4 Decristofaro MF, Betz BL, Rorie CJ, Reisman DN, Wang W and Weissman BE: Characterization of SWI/SNF protein expression in human breast cancer cell lines and other malignancies. J Cell Physiol 186: 136-145, 2001.

5 Sun $\mathrm{H}$, Zhong X, Wang C, Wang S, Lin L, Zou R, Wu Y, Sun $\mathrm{N}$, Sun G, Wen T, Chi ZH and Zhao Y: SNF5 is involved in 
suppression of hepatocellular carcinoma progression via TGFbeta 1 signaling. Anat Rec 299: 869-877, 2016.

6 Terracciano LM, Glatz K, Mhawech P, Vasei M, Lehmann FS, Vecchione R and Tornillo L: Hepatoid adenocarcinoma with liver metastasis mimicking hepatocellular carcinoma: An immunohistochemical and molecular study of eight cases. Am J Surg Pathol 27: 1302-1312, 2003.

7 Ushiku T, Shinozaki A, Shibahara J, Iwasaki Y, Tateishi Y, Funata $\mathrm{N}$ and Fukayama M: SALL4 represents fetal gut differentiation of gastric cancer, and is diagnostically useful in distinguishing hepatoid gastric carcinoma from hepatocellular carcinoma. Am J Surg Pathol 34: 533-540, 2010.
8 Ushiku T, Uozaki H, Shinozaki A, Ota S, Matsuzaka K, Nomura S, Kaminishi M, Aburatani H, Kodama T and Fukayama M: Glypican 3-expressing gastric carcinoma: Distinct subgroup unifying hepatoid, clear-cell, and alpha-fetoprotein-producing gastric carcinomas. Cancer Sci 100: 626-632, 2009.

Received October 25, 2018

Revised November 1, 2018

Accepted November 2, 2018 